\title{
Ultrathin phase-change coatings on metals for electrothermally tunable colors
}

\author{
Gokhan Bakan, ${ }^{1,2, a)}$ Sencer Ayas, ${ }^{2, b)}$ Tohir Saidzoda, ${ }^{1}$ Kemal Celebi, ${ }^{2}$ and Aykutlu Dana ${ }^{2}$ \\ ${ }^{1}$ Deparment of Electrical and Electronics Engineering, Antalya International University, Antalya 07190, \\ Turkey \\ ${ }^{2}$ UNAM Institute of Materials Science and Nanotechnology, Bilkent University, Ankara 06800, Turkey
}

(Received 21 June 2016; accepted 6 August 2016; published online 18 August 2016)

\begin{abstract}
Metal surfaces coated with ultrathin lossy dielectrics enable color generation through strong interferences in the visible spectrum. Using a phase-change thin film as the coating layer offers tuning the generated color by crystallization or re-amorphization. Here, we study the optical response of surfaces consisting of thin (5-40 nm) phase-changing $\mathrm{Ge}_{2} \mathrm{Sb}_{2} \mathrm{Te}_{5}$ (GST) films on metal, primarily $\mathrm{Al}$, layers. A color scale ranging from yellow to red to blue that is obtained using different thicknesses of as-deposited amorphous GST layers turns dim gray upon annealing-induced crystallization of the GST. Moreover, when a relatively thick $(>100 \mathrm{~nm})$ and lossless dielectric film is introduced between the GST and Al layers, optical cavity modes are observed, offering a rich color gamut at the expense of the angle independent optical response. Finally, a color pixel structure is proposed for ultrahigh resolution (pixel size: $5 \times 5 \mu \mathrm{m}^{2}$ ), non-volatile displays, where the metal layer acting like a mirror is used as a heater element. The electrothermal simulations of such a pixel structure suggest that crystallization and re-amorphization of the GST layer using electrical pulses are possible for electrothermal color tuning. Published by AIP Publishing.

[http://dx.doi.org/10.1063/1.4961368]
\end{abstract}

Strong absorption of light can be achieved through strong interference effects enabled by nanometers thick lossy dielectrics on reflective layers such as metals. Recently, such surfaces have been investigated for color generation/printing, ${ }^{1-4}$ enhanced light absorption in the visible ${ }^{5}$ through Ge or Si coating of metals like $\mathrm{Au}$ and $\mathrm{Ag}$, and infrared (IR) filters $^{6}$ and emitters ${ }^{7}$ using thin $\mathrm{VO}_{2}$ on sapphire. Moreover, phase-changing $\mathrm{Ge}_{2} \mathrm{Sb}_{2} \mathrm{Te}_{5}$ (GST) films on Au are demonstrated for enhanced optical data storage using the same optical phenomenon. ${ }^{8}$ GST shows reversible phase transitions between amorphous and crystalline states accompanied by significant changes in its optical and electrical properties. Hence, GST and other phase-change materials are commonly used for optical ${ }^{9,10}$ and electrical ${ }^{11,12}$ data storage media. Amorphous to crystalline transition is typically achieved by annealing the material below the melting temperature for at least tens of nanoseconds. ${ }^{13}$ The reverse transition requires melting and quenching the material which can be achieved in a much shorter time scale $(\sim \mathrm{fs}){ }^{8,14}$ External heating ${ }^{15,16}$ or self-heating using lasers ${ }^{17,18}$ or electrical pulses ${ }^{19,20}$ are used for annealing or melt-quenching. Recently, GST has found its way into visible ${ }^{21}$ and infrared plasmonic absorber surfaces using $\mathrm{Al}$ nano-antennas ${ }^{14,22}$ and Au nanopatches. ${ }^{23,24}$ The optical response of the plasmonic infrared (IR) absorber surfaces is tuned by annealing on a hot plate ${ }^{22}$ or with a train of low-power femtosecond pulses $^{14}$ for amorphous to crystalline transition and a single high-power femtosecond laser pulse for crystalline to amorphous

\footnotetext{
a) gokhan.bakan@antalya.edu.tr

b) Present address: Bio-Acoustic-MEMS in Medicine (BAMM) Laboratory, Department of Radiology, Canary Center at Stanford for Cancer Early Detection, Stanford University School of Medicine, Palo Alto, California 94304, USA.
}

transition. ${ }^{14}$ Very recently, electro-thermally tunable pixel structures consisting of a Pt/ITO/GST/ITO stack have been demonstrated using Fabry-Perot resonances. ${ }^{19}$

Here, we study optical responses of surfaces formed by ultrathin $(5-40 \mathrm{~nm})$ GST coated metal mirrors, primarily Al, and investigate their potential application as tunable color pixels for large area displays. The surfaces studied here have a simple 2-layer structure consisting of continuous thin GST films on $80 \mathrm{~nm}$ thick Al. The optical responses of the surfaces are tuned by annealing samples on a hot plate $\left(\mathrm{T}>150^{\circ} \mathrm{C}\right.$ ) as the GST film morphology changes from the as-deposited amorphous to face-centered cubic (fcc) crystalline phase. ${ }^{14,22,25}$ Electrothermal simulations of a small pixel made of such GST structures are also performed to assess the possibility of achieving thermally tunable large-area displays by electrical heating. In the proposed pixel structure, the mirror layer also functions as a heater in contrast to selfheating of the GST layer in the Pt/ITO/GST/ITO stack demonstrated in Ref. 19. As a result, the proposed structure allows larger $(>\mu \mathrm{m})$ pixels and hence enables large-area fabrication; whereas the Pt/ITO/GST/ITO stack can offer richer color gamut.

The most commonly used metals for the plasmonic applications, i.e., $\mathrm{Au}, \mathrm{Ag}$, and $\mathrm{Al}$, are considered for this study (see Figure S1 in the supplementary material for the optical constants). The initial simulation results for $20 \mathrm{~nm}$ amorphous GST (aGST) on optically thick Au, Ag, and Al metal layers reveal reflection minimum at $\sim 640 \mathrm{~nm}$ for $\mathrm{Au}$ and $\mathrm{Ag}$, and $\sim 400 \mathrm{~nm}$ for $\mathrm{Al}$ (Figure 1(a)). These results show that $\mathrm{Al}$ enables absorptions centered around the ultraviolet (UV) wavelengths for thin film thickness values which can be reliably deposited by a sputtering system. Furthermore, the surface colors achieved using Ag mirrors 


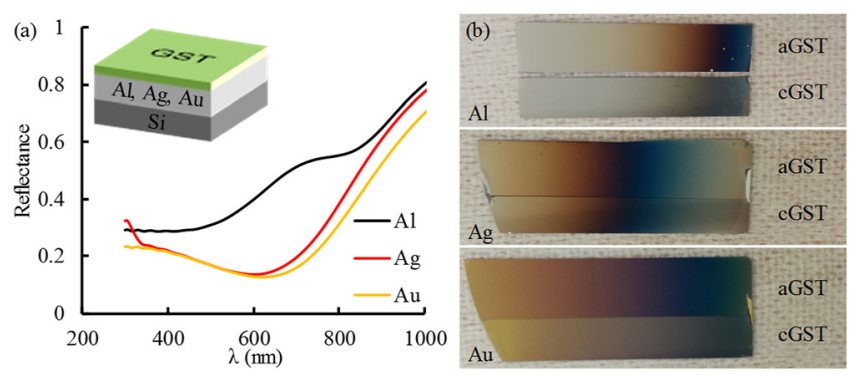

FIG. 1. GST coated metal surfaces. (a) Simulated reflection spectra of $20 \mathrm{~nm}$ aGST on $80 \mathrm{~nm} \mathrm{Al}, \mathrm{Ag}$, and $\mathrm{Au}$ layers. (Inset) Illustration of the structures. (b) Color gradients on surfaces achieved by depositing GST films with increasing thickness from left $(<5 \mathrm{~nm})$ to right $(>50 \mathrm{~nm})$ on $\mathrm{Al}, \mathrm{Ag}$, and $\mathrm{Au}$ layers. Thermal color tuning is achieved only on the $\mathrm{Al}$ and $\mathrm{Au}$ bottom layers.

cannot be tuned by crystallization of the top GST layer (Figure 1(b), Figure S2 in the supplementary material). Hence, $\mathrm{Al}$ is preferred over Au due to its low cost and reflection minima achieved at lower wavelengths despite its interband absorption at $\sim 800 \mathrm{~nm}^{26}$ The colors generated on the $\mathrm{Au}$ mirror is found to be brighter compared to what is reported by Schlich et al. ${ }^{8}$ using the $\mathrm{Au} / \mathrm{Ti} / \mathrm{TiN} / \mathrm{GST} / \mathrm{SiO}_{2}$ stack. This is attributed to the thin $(5 \mathrm{~nm}) \mathrm{Ti} / \mathrm{TiN}$ barrier layer that Schlich et al. ${ }^{8}$ used between the Au and GST layer.

The top GST thickness is varied from 5 to $140 \mathrm{~nm}$ to observe the reflection minimum to shift from the UV to near IR regimes (Figure S3 in the supplementary material). Colors of the surfaces can be tuned from yellow to red to blue by varying the aGST thickness from 5 to $40 \mathrm{~nm}$ (Figures 1(b) and 2(a)). The surfaces with as-deposited aGST films are annealed beyond $150^{\circ} \mathrm{C}$ using $\sim 1 \mathrm{~K} / \mathrm{s}$ heating rates on a hot plate for color tuning. The surfaces look dim gray for all GST thicknesses upon crystallization (Figure 2(a)). The reflection spectra measurements reveal that the observed colors are due to absorption of certain portions of the visible regime (Figure 2(b)). The reflection spectra change upon crystallization resulting in almost constant low-reflectance in the visible regime, which is responsible for the dim gray colors. For the $20 \mathrm{~nm}$ GST film, the absorption wavelength to the film thickness ratio $(\lambda / \mathrm{t})$ is $7.4 n$ for amorphous and $8 n$ for fcc phases of GST, both being larger than $4 n$ which is observed for conventional interference coatings. The $\lambda / \mathrm{t}$ ratio decreases with increasing absorption wavelength, being close to $4 \mathrm{n}$, owing to decreasing extinction coefficients of aGST and crystalline GST (cGST) (Figure S1 in the supplementary material). aGST behaves like a lossless dielectric $(k=0)$ beyond $\lambda \sim 1.5 \mu \mathrm{m}$, resulting in weak absorptions as opposed to the strong absorptions obtained in the visible regime (Figure S4 in the supplementary material). The extinction coefficient of cGST is never zero due to the Drude contribution in the near and mid-IR. ${ }^{25}$ The reflection minimum in the near IR obtained using thicker (80-140 nm) aGST films consistently red-shifts and exhibits almost perfect absorptions upon crystallization because of larger $n$ and non-zero $k$ of cGST in this regime (Figure S4 in the supplementary material). The thermally tunable optical behavior of the surfaces in the near and mid-infrared regimes offers further photonics applications such as active spectrally selective infrared absorbers/emitters and active electric field enhancement platforms for sensitive infrared absorption spectroscopy. Simulation results using the measured complex refractive index of aGST and cGST capture the measured reflection spectra (Figure 2(c)). Figure S5 in the supplementary material shows simulation results with $5 \mathrm{~nm}$ GST thickness increment providing better visualization of tuning of the reflection spectra upon crystallization of the GST film.

The as-fabricated GST films are in the amorphous phase. The GST phase transitions to fcc $\sim 150^{\circ} \mathrm{C}$ while annealing the films on a hot plate with relatively low heating rates $(\sim 1 \mathrm{~K} / \mathrm{s})$. Further increment in the temperature switches the phase from fcc to hexagonal $\sim 200^{\circ} \mathrm{C}$. These transition temperatures depend on the heating rate and the stress on the GST film. ${ }^{27,28}$ Crystallization of the top GST film while annealing can also be monitored by visually inspecting the surface color. The temperature coefficient of the refractive

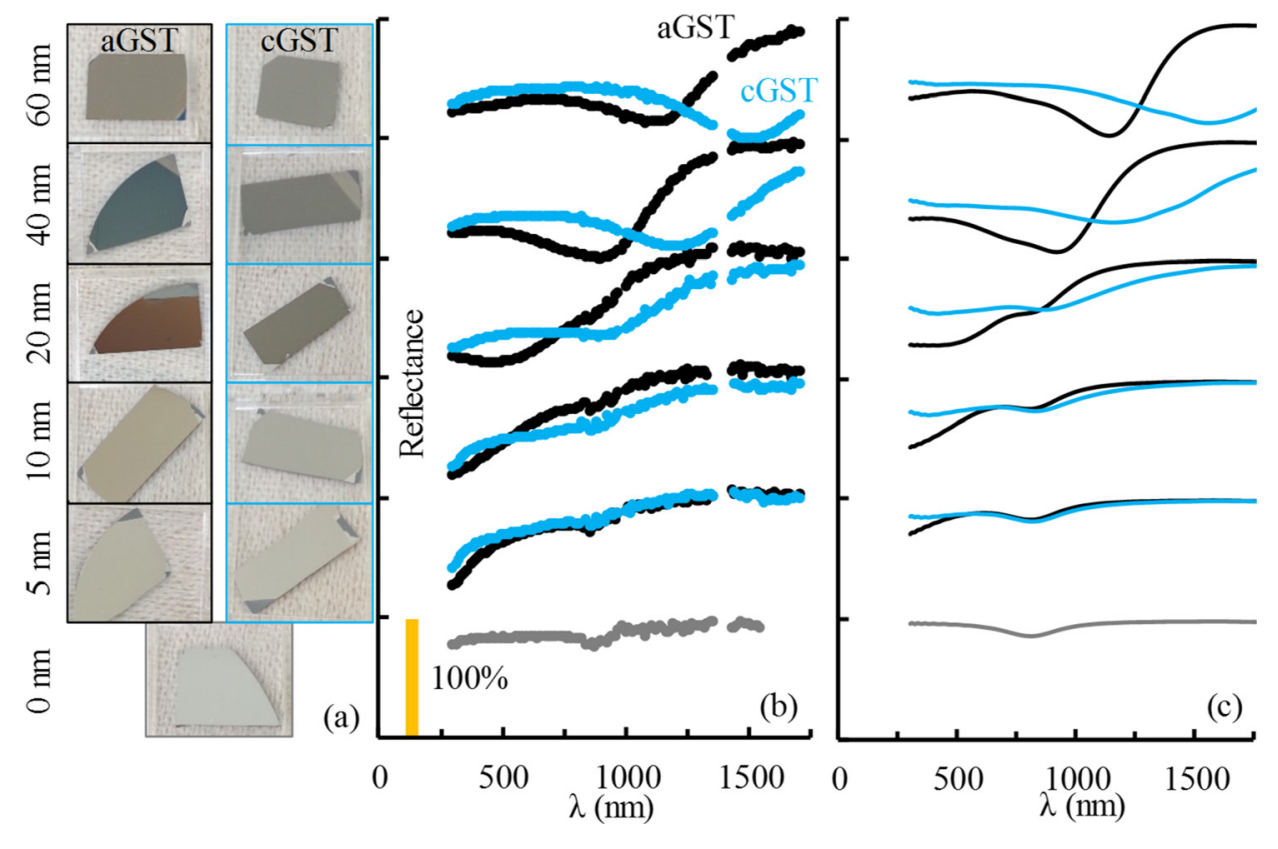

FIG. 2. Optical properties of GST coated Al surfaces. (a) Photographs of GST films with indicated thicknesses on $80 \mathrm{~nm} \mathrm{Al}$. Corresponding (b) measured (c) simulated reflection spectra. Reflection spectra scales between $0 \%$ and $100 \%$. The curves are shifted along the $y$-axis. The crystallized films are expected to be $5 \%$ thinner than the as-fabricated aGST films. 
index for GST is reported to be small $\left(\Delta \mathrm{n} / \Delta \mathrm{T}=-2.5 \times 10^{-3}\right.$ $1 / \mathrm{K}, \Delta \mathrm{k} / \Delta \mathrm{T}=6.4 \times 10^{-4} 1 / \mathrm{K}$ at $\left.600 \mathrm{~nm}\right),{ }^{29}$ and hence no significant color change is observed until the amorphous to fcc-phase transition. Once the aGST films start crystallizing, the colors of the surfaces suddenly turn dim gray and stay that way as the surfaces cool down to the room temperature. Further annealing the surfaces beyond $200^{\circ} \mathrm{C}$ changes the GST phase to hexagonal, which has an indistinguishable effect on the reflection spectra (Figures 3(a) and 3(b)) due to small difference in the refractive indices of GST in fcc and hexagonal-phases. ${ }^{29}$

The reflection spectra and colors of the surfaces are unaffected up to high angles of incidence $\left(\sim 65^{\circ}\right)$ (Figures 3(c) and 3(d)) owing to extremely thin GST layers. Similar results are observed with other strong interference surfaces such as $15 \mathrm{~nm}$ Ge coated Au surfaces. ${ }^{3}$ Owing to the angleindependent response of ultrathin coatings, flexible, bendable, and rough substrates such as $\mathrm{Al}$ foil (Figure S6 in the supplementary material) or paper $^{4}$ can be used for the strong interference applications.

The color gamut that can be achieved with ultrathin coating of metal mirrors can be enhanced introducing a lossless film between the mirror and absorber layers (Figure 4(a)). A similar multi-layer structure, specifically a thin $(7 \mathrm{~nm})$ GST layer sandwiched between two ITO layers on top of a Pt mirror, has recently been used by Hosseini et al. ${ }^{19}$ for color tuning. Another recent study uses such a multilayer structure with 2 GST layers separated by a thermal barrier layer. ${ }^{30}$ In the study by Hosseini et al., the bottom ITO layer thickness has been varied to adjust the color, which is also further varied by changing the GST phase using self-heating through electrical pulses. Such electrical heating requires the use of a transparent conductive material (ITO) instead of a lossless dielectric. In contrast, instead of using an ITO layer, here we use an $\mathrm{HfO}_{2}$ layer between the top GST and bottom Al layers which enables the metal layer function as a heater as well. Optical simulation results show that a $\mathrm{HfO}_{2}$ layer on top of $\mathrm{Al}$ without a GST top layer shows weak resonances in the visible regime (Figure 4(a)). A $10 \mathrm{~nm}$ aGST layer on top of $\mathrm{HfO}_{2}$ induces strong resonances which broaden and red-shift with increasing $\mathrm{HfO}_{2}$ thickness $(0-120 \mathrm{~nm})$ and totally disappear for thicker $\mathrm{HfO}_{2}$ (Figure
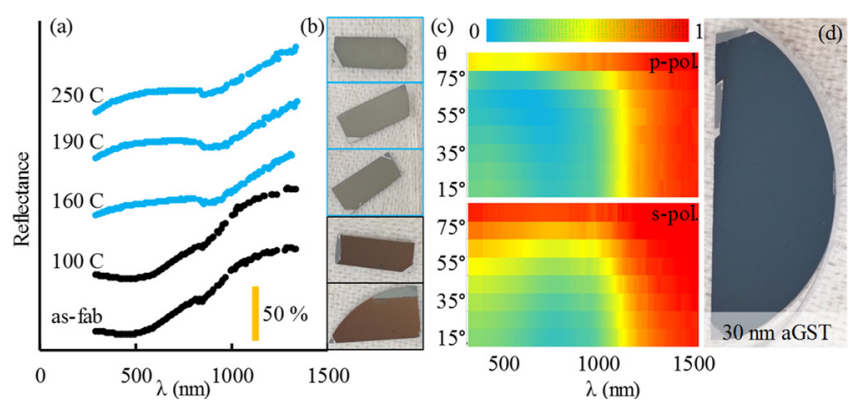

FIG. 3. The effect of annealing temperature and angle of incidence on the optical response of the surfaces. (a) Measured reflection spectra of $20 \mathrm{~nm}$ GST coated Al surfaces after annealing with a heating rate of $\sim 1 \mathrm{~K} / \mathrm{s}$ up to the temperatures shown next to each curve. The curves are shifted along the y-axis. (b) Photographs of the surfaces. (c) Measured reflection spectra of the $30 \mathrm{~nm}$ aGST coated $\mathrm{Al}$ surface as the angle of incidence is varied from $15^{\circ}$ to $85^{\circ}$ with $10^{\circ}$ steps for $\mathrm{p}$ - and s-polarizations. (d) Photograph of the surface with $30 \mathrm{~nm}$ aGST.
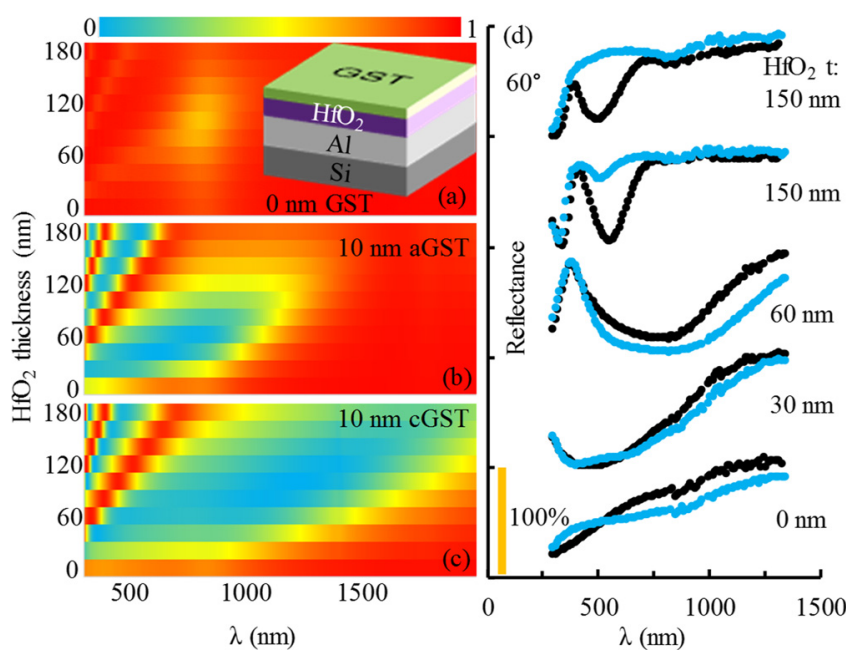

FIG. 4. The effect of the $\mathrm{HfO}_{2}$ layer between GST and Al layers. Calculated reflection spectra for varying thickness of $\mathrm{HfO}_{2}$ on $\mathrm{Al}$ (a) without GST and with (b) $10 \mathrm{~nm}$ aGST and (c) $10 \mathrm{~nm}$ cGST top layers. (Inset) Illustration of the structure. (d) Measured reflection spectra of $10 \mathrm{~nm}$ aGST (black) and $10 \mathrm{~nm}$ cGST (blue) top layers on $\mathrm{HfO}_{2}$ films with indicated thicknesses. The curves are shifted along the $y$-axis. The angle of incidence for the top curves is $60^{\circ}$ and $30^{\circ}$ for the rest.

4(b)). Crystallization of the GST layer also broadens the resonances for thin $\mathrm{HfO}_{2}$ (Figure 4(c)). Fabry-Pérot resonances appear for further increment in the $\mathrm{HfO}_{2}$ thickness $(>80 \mathrm{~nm}) .{ }^{19,31,32}$ The absorption bands achieved with FabryPérot resonances are narrower than those observed for strong interference effects, resulting in brighter surfaces colors. The blue-shift in the Fabry-Pérot resonances with crystallization of the top GST layer shifts the surface color to a different tone instead of a complete color change observed for the surfaces without a spacer layer. The measured reflection spectra show the broadening and red-shift of the reflection minimum and further broadening with increasing $\mathrm{HfO}_{2}$ thickness $(0-60 \mathrm{~nm})$, as well as the emergence of the Fabry-Pérot resonances for $150 \mathrm{~nm} \mathrm{HfO}_{2}$ (Figure 4(d)). The reflection spectra and hence the colors of the surfaces with thick $\mathrm{HfO}_{2}$ layers significantly depend on the angle of incidence as shown by the reflection measurements performed at $30^{\circ}$ and $60^{\circ}$ (Figure 4(d)) and the photographs taken at low and high viewing angles (Figure S7 in the supplementary material). The lossless dielectric layer between the GST and metal layers could also function as a barrier to prevent diffusion of the metal into GST, which is a great concern when chemically active metals are used in contact with GST.

The observed color gradient by changing either the GST or the dielectric thickness and color change with crystallization of the GST layer led us to study its potential for high resolution, nonvolatile, reflective displays. Both amorphous to crystalline and crystalline to amorphous phase transitions are required for a rewritable display. While simply annealing the films is enough for crystallization as presented here, amorphization requires melting and quenching the material which is typically achieved using a single femtosecond laser pulse at the film level. ${ }^{8}$ However, to form a display it is desired that the phase of the GST film, hence the surface color, is tuned using electrical pulses, similar to the demonstration of tiny displays (pixel size: $300 \times 300 \mathrm{~nm}^{2}$ ) formed by the ITO/GST/ ITO/Pt stack of Hosseini et al. ${ }^{19}$ Such dimensions, however, 
do not allow large area displays as they require e-beam lithography or projection photolithography for patterning. Simply scaling up the tiny pixels formed by sandwiched GST layer for large area displays would not work, since small filaments forming in the GST layer carry most of the electrical current during amorphization pulses resulting in heating and melting of small spots in the GST layer. To uniformly heat and melt the GST layer for uniform amorphization, we propose the bottom reflector to function also as a heater (Figure $5(\mathrm{a})$ ). If the electrical contacts are designed to be thicker than the metal layer $(\sim 100 \mathrm{~nm})$, most of the electrical power is expected to be dissipated in the metal layer under the GST film. The proposed structure with $5 \times 5 \mu \mathrm{m}^{2}$ area is simulated to test its heating and cooling behavior using the electrothermal (Joule heating) module of COMSOL Multiphysics. The simulation details are provided in the methods section and Figure S9 in the supplementary material. The simulated structure includes a $100 \mathrm{~nm} \mathrm{SiO}$ capping layer which aims to prevent evaporation and deformation of the GST layer during melting. Alternatively, the structures can be formed by depositing the GST film on a glass substrate followed by the metal deposition to protect the GST layer (Figure S8 in the supplementary material). In this case, the surfaces show similar reflection spectra with a slight red-shift. The simulated structure uses Au with a higher melting temperature instead of Al. The simulation results suggest that both crystallization and melting temperatures can be achieved using appropriate electrical pulses (Figure 5(b)). Amorphization requires quenching after melting which necessitates the cooling time constants to be short, and hence the pixel structures to be small. The results show that for the modelled pixel size $\left(5 \times 5 \mu \mathrm{m}^{2}\right)$, short heating/cooling time constants of $\sim 100$ ns can be realized (Figure 5(c)). Although, the GST volume which needs to be annealed for this operation is larger than typical PCM cells, SET and RESET operations have been demonstrated for even larger scale phase-change RF switches. ${ }^{33}$ The proposed color pixel structure is tested by fabricating an array of Al wires with small dimensions $\left(2 \times 2 \mu \mathrm{m}^{2}\right)$ and coating them with $20 \mathrm{~nm}$ aGST. The initial surface color is tuned by passing electric current $(100 \mathrm{~mA})$ through the wires and annealing the GST patch on top of the wires (Figure S10 in the supplementary material). The non-uniform temperature distribution and high current levels estimated by the measurements and simulations can be overcome with smart designs
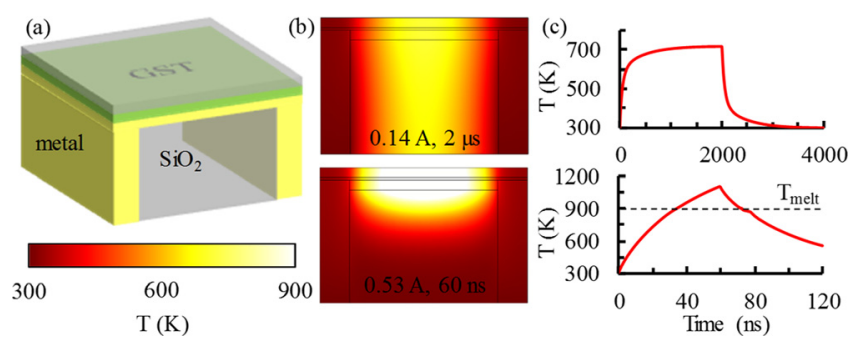

FIG. 5. Electrothermal simulation results. (a) Illustration of the simulated pixel structure. $\mathrm{SiO}_{2}$ layers are shown semi-transparent for clarity. (b) Temperature distributions on the 2D structure at the end of electrical pulses applied for crystallization (top) and amorphization (bottom). (c) Evolution of the peak temperature in the GST film during and following the crystallization and amorphization pulses. and materials, such as serpentine heater structures, highresistivity reflective materials, and better thermal isolation.

In summary, we observe that thin GST layers on metals, such as $\mathrm{Al}, \mathrm{Ag}$, and $\mathrm{Au}$ produce colors owing to strong interference effects in the visible. A color scale ranging from yellow to red to blue that is produced with different thicknesses of GST on Al layers changes to dim gray upon crystallization of the GST film by annealing. The surfaces show angleindependent optical responses as confirmed by the reflection measurements and perceived by the naked eye. Introducing a lossless dielectric $\left(\mathrm{HfO}_{2}\right)$ layer between the metal and GST layers enables further tuning of the optical response by exciting the Fabry-Pérot modes. The narrow Fabry-Pérot resonances which can be tuned by the dielectric thickness offer a rich color gamut which can be shifted by crystallization of the GST layer. Finally, the possibility of forming a display is assessed through electrothermal modeling of a pixel structure consisting of $\mathrm{SiO}_{2} / \mathrm{GST} /$ metal stack. The metal layer acts as a reflector as well as a heater for uniform heating of the atop GST film. Simulation results for a pixel element with $20 \mathrm{~nm}$ GST on $80 \mathrm{~nm}$ metal layer and $5 \times 5 \mu \mathrm{m}^{2}$ area show that the temperature levels and quenching time required for amorphization can be achieved. Such a pixel structure capable of thermal color tuning can enable highresolution, non-volatile, large-area, rewritable displays.

See supplementary material for fabrication, measurements, and simulations details, optical constants of the materials, additional optical measurement results, and demonstration of a color pixel.

This work was partially supported by TUBITAK Grant No. 114E960 and EU FP7:People-IAPP NanoBacterPhageSERS.

${ }^{1}$ E. Gaul, J. Chem. Educ. 70, 176 (1993).

${ }^{2}$ F. F. Schlich and R. Spolenak, Appl. Phys. Lett. 103, 213112 (2013).

${ }^{3}$ M. A. Kats, R. Blanchard, P. Genevet, and F. Capasso, Nat. Mater. 12, 20 (2013).

${ }^{4}$ M. A. Kats and F. Capasso, Appl. Phys. Lett. 105, 131108 (2014).

${ }^{5}$ K.-T. Lee, S. Seo, J. Y. Lee, and L. J. Guo, Adv. Mater. 26, 6324 (2014).

${ }^{6}$ M. A. Kats, D. Sharma, J. Lin, P. Genevet, R. Blanchard, Z. Yang, M. M. Qazilbash, D. N. Basov, S. Ramanathan, and F. Capasso, Appl. Phys. Lett. 101, 221101 (2012).

${ }^{7}$ M. A. Kats, R. Blanchard, S. Zhang, P. Genevet, C. Ko, S. Ramanathan, and F. Capasso, Phys. Rev. X 3, 041004 (2013).

${ }^{8}$ F. F. Schlich, P. Zalden, A. M. Lindenberg, and R. Spolenak, ACS Photonics 2, 178 (2015).

${ }^{9}$ A. V. Kolobov, P. Fons, A. I. Frenkel, A. L. Ankudinov, J. Tominaga, and T. Uruga, Nat. Mater. 3, 703 (2004).

${ }^{10}$ M. Wuttig and N. Yamada, Nat. Mater. 6, 824 (2007).

${ }^{11}$ D. Ielmini and A. L. Lacaita, Mater. Today 14, 600 (2011).

${ }^{12}$ S. Raoux, F. Xiong, M. Wuttig, and E. Pop, MRS Bull. 39, 703 (2014).

${ }^{13}$ M. Wuttig and M. Salinga, Nat. Mater. 11, 270 (2012).

${ }^{14}$ A.-K. U. Michel, P. Zalden, D. N. Chigrin, M. Wuttig, A. M. Lindenberg, and T. Taubner, ACS Photonics 1, 833 (2014).

${ }^{15}$ R. Jeyasingh, S. W. Fong, J. Lee, Z. Li, K.-W. Chang, D. Mantegazza, M. Asheghi, K. E. Goodson, and H.-S. P. Wong, Nano Lett. 14, 3419 (2014).

${ }^{16}$ J. Orava, A. L. Greer, B. Gholipour, D. W. Hewak, and C. E. Smith, Nat. Mater. 11, 279 (2012).

${ }^{17}$ V. Weidenhof, I. Friedrich, S. Ziegler, and M. Wuttig, J. Appl. Phys. 89, 3168 (2001).

${ }^{18}$ M. K. Santala, B. W. Reed, S. Raoux, T. Topuria, T. LaGrange, and G. H. Campbell, Appl. Phys. Lett. 102, 174105 (2013).

${ }^{19}$ P. Hosseini, C. D. Wright, and H. Bhaskaran, Nature 511, 206 (2014).

${ }^{20}$ A. Sebastian, M. Le Gallo, and D. Krebs, Nat. Commun. 5, 4314 (2014). 
${ }^{21}$ T. Cao, C. Wei, R. E. Simpson, L. Zhang, and M. J. Cryan, Sci. Rep. 4, 3955 (2014).

${ }^{22}$ A.-K. U. Michel, D. N. Chigrin, T. W. W. Maß, K. Schönauer, M. Salinga, M. Wuttig, and T. Taubner, Nano Lett. 13, 3470 (2013).

${ }^{23}$ T. Cao, L. Zhang, R. E. Simpson, and M. J. Cryan, J. Opt. Soc. Am. B 30, 1580 (2013).

${ }^{24}$ Y. Chen, X. Li, X. Luo, S. A. Maier, and M. Hong, Photonics Res. 3, 54 (2015).

${ }^{25}$ K. Shportko, S. Kremers, M. Woda, D. Lencer, J. Robertson, and M. Wuttig, Nat. Mater. 7, 653 (2008).

${ }^{26}$ S. Ayas, A. E. Topal, A. Cupallari, H. Güner, G. Bakan, and A. Dana, ACS Photonics 1, 1313 (2014).

${ }^{27}$ J. L. M. Oosthoek, K. Attenborough, G. A. M. Hurkx, F. J. Jedema, D. J. Gravesteijn, and B. J. Kooi, J. Appl. Phys. 110, 024505 (2011).
${ }^{28}$ H. K. Peng, K. Cil, A. Gokirmak, G. Bakan, Y. Zhu, C. S. Lai, C. H. Lam, and H. Silva, Thin Solid Films 520, 2976 (2012).

${ }^{29}$ M. Kuwahara, O. Suzuki, N. Taketoshi, Y. Yamakawa, T. Yagi, P. Fons, K. Tsutsumi, M. Suzuki, T. Fukaya, J. Tominaga, and T. Baba, Jpn. J. Appl. Phys., Part 1 45, 1419 (2006).

${ }^{30}$ S. Yoo, T. Gwon, T. Eom, S. Kim, and C. S. Hwang, ACS Photonics 3, 1265 (2016).

${ }^{31}$ Z. Li, S. Butun, and K. Aydin, ACS Photonics 2, 183 (2015).

${ }^{32}$ M. Serhatlioglu, S. Ayas, N. Biyikli, A. Dana, and M. E. Solmaz, Opt. Lett. 41, 1724 (2016).

${ }^{33}$ N. El-Hinnawy, P. Borodulin, B. Wagner, M. R. King, J. S. Mason, E. B. Jones, S. McLaughlin, V. Veliadis, M. Snook, M. E. Sherwin, R. S. Howell, R. M. Young, and M. J. Lee, IEEE Electron Device Lett. 34, 1313 (2013). 